\title{
An Optimal Exiting Time for Old Generation Product in Supply Chain
}

\author{
Yu Qi ${ }^{*}$, Yong Luo \\ Industrial Technology Research Institute, Zhengzhou University, Zhengzhou, China \\ Email address: \\ qy979340369@163.com (Yu Qi), luoyong@zzu.edu.cn (Yong Luo) \\ ${ }^{*}$ Corresponding author
}

\section{To cite this article:}

Yu Qi, Yong Luo. An Optimal Exiting Time for Old Generation Product in Supply Chain. American Journal of Operations Management and Information Systems. Vol. 4, No. 1, 2019, pp. 16-25. doi: 10.11648/j.ajomis.20190401.12

Received: February 12, 2019; Accepted: March 25, 2019; Published: April 18, 2019

\begin{abstract}
I) Brief problem description: With technology developing, many series products which have several generations appear in one market. The sales of new and old generations of a product in one market influences each other. (II) The study aim: In order to help the supply chain to obtain more benefits, this paper makes a study on the exiting of old generation products. (III) Method: Based on Fisher model and multi-generation product diffusion model, this paper establishes an optimal decision-making model for the exiting time of old generation product, and analyzes the relationship between the optimal exiting time of the old generation product and the technical level of the new generation product. (IV) Result and conclusion: Observations from this study include (1) when the profit of the new generation product sold only and that of the two generations sold simultaneously are the same, it is the optimal time for old generation product to exit market, and (2) the higher the technical level of new generation product is, the earlier old generation product will exit from market, and the more profit supply chain will get. (V) Significance: The research result will help some company make optimal decision-making for the exiting time of old generation product, and get more profit.
\end{abstract}

Keywords: Optimal Decision, Multi-generation Product, Product Exit, Exiting Time

\section{Introduction}

The rapid development of science and technology makes market more competitive and accelerates the frequency of product innovation. Product upgrade is a special product innovation process which develop a new generation product by modifying an old generation product. Especially, in some high-tech electronic product industries, such as computer, cell phone, companies constantly develop updated generation product to meet differentiated consumer demand, thereby occupying as much share of market as possible and gaining profits.

The sale of multiple generations of products in one market is affected by each other. With continuous introduction of improved new generations products, it is necessary to consider the exit of old generation product in order to maximize the supply chain profit. When old generation product is sold in market for a long time at a relatively low price, considering the price/performance ratios of two generations products, consumers will tend to buy old products, thus affecting the sales of new generation product and supply chain profit. It is also unsuitable for old generation product to exit from market as soon as improved new generation product is introduced. Because consumers may not yet accept new generation product, which is not helpful for the sales and diffusion of new products, it is necessary to consider and study the exit of old products from the market. Before analyzing the product exit, we need to learn something about the diffusion of the product.

Firstly, some studies on single product diffusion are reviewed. In 1969, the Bass model proposed by Frank Bass [1] considered the innovator diffusion model and the imitator diffusion model. Bass model is a classical single generation product diffusion model, which plays an important role in the field of product diffusion. and was used widely to study new product diffusion. In 1971, the technological innovation diffusion model proposed by Fisher [2] is also an abstract description of the diffusion of new products. Through a series of empirical studies, the market share changes of new products are given. This technological innovation diffusion model is also known as Fisher model. In 2006, Shaikh and Rangaswa [15] pointed out that the reception process of innovative products in social networks can be described by network models. Based on these single-generation product diffusion 
models, many scholars have carried out many researches. Based on Bass [1] and Luigi [14], Quan [8] studied the stackelberg game of new product's price between manufacturers and sellers. Gupta Manak C. [9] established theoretical conditions for optimal pricing and advertising policy using a dynamic model. Luo [11] studied the process of product renewal in a supply chain, and built a market share shift model for renewal product based on a increment function and a shift function. Lu [16] accords to the optimal control theory, adds competitive product's price and advertising investment are into Bass model, establishes the new product diffusion control model to study the optimal product diffusion process, and gets the conclusion that the diffusion mechanism of new products presents a complex nonlinear feature.

With the development of science and technology, the market competition intensifies. In order to meet the needs of consumers, the supply chain needs to continuously launch new products, which will lead to the coexistence of multiple generations of products in the market. Most research, however, has only paid close attention to the single product diffusion. Therefore, in 1987, on the basis of the Bass model, Norton J A and Bass F M [3] proposed the Norton-Bass model to study the diffusion of multi-generation replacement products. Luo [4] developed a two-stage differentiated products supply chain model based on Fisher model. Seref Michelle M. H. [5] considered both the timing and dynamic pricing decisions to determine an appropriate entry strategy of new product into the marketplace. Kenji [6] investigated the optimal timing and level of wholesale and retail prices set in multi-channel supply chains. Chanda [7] established a mathematical model of Economic Order Quantity (EOQ) for two-generation substitutable product range. The model was based on the assumption that technological advancements do not essentially imply that existing generation products will be withdrawn from the market immediately. Druehl [10] studied the optimal speed for companies to introduce new products regularly based on the multi-generation Bass model of Norton and Bass. Kalish [18] studied the monopolistic pricing with dynamic demand and production cost.

If multi-generation products are sold in the market, the diffusion of new and old generation products will affect each other. At present, there is a lack of research on the diffusion of old generation products. Argouslidis [12] studied the speed of product exit, but could not determine the specific time for old generation product to exit. Lim [13] developed an analytical model to analyze the profits associated with two product rollover strategies, discussed the optimal time and price to introduce a new generation product and eliminate an old generation product. However, there is no simulation to verify the accuracy of its model.

In general, the research on product diffusion mainly focuses on the new product diffusion and multi-product diffusion. Research on old products in the current literature is not enough. Therefore, based on the single-generation and multi-generation product diffusion models constructed by Fisher model, this paper further constructs the exiting model of old products and further studies the exiting time of old products through simulation analysis.

\section{Supply Chain and Sales Model}

\subsection{Multi-generation Product Supply Chain Model}

In general, products on the market go from production to sales, go through suppliers and retailers, and eventually reach the market for sales. There are only two links in the supply chain: wholesale and retail, that is, the supply chain contains only two parts: supplier and retailer. Since this paper studies the profits of the supply chain from each generation products, it is the sum of the profit obtained from the two parts from each generation of products. At this point, a simplified supply chain model can be constructed as Figure 1 .

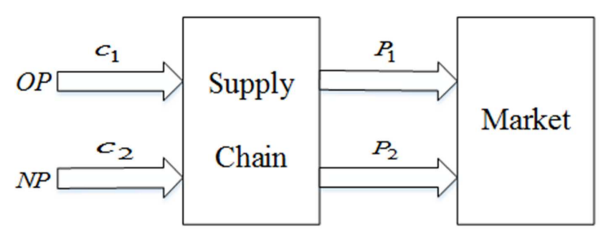

Figure 1. Supply chain simplified model.

In the model, it has two generations of a product in a same time period. The operation of the supply chain is as follows: firstly, the supply chain sells the old generation of a product (OP) to the market at a retail price $P_{1}$; at the same time, the new generation of the product (NP) is developed by upgrading the OP. The supply chain sells the NP to the market at a retail price $P_{2}$. The costs to the supply chain for the two generations are $c_{1}$ and $c_{2}$, respectively, and the cost to the supply chain to sell each of the two generations is $c$, which is the same for both OP and NP. The supply chain wants to maximize its profits.

\subsection{Two-generation Products Sales Model}

With regard to the diffusion of new products under the influence of scientific and technological progress, Fisher [2] proposed a substitution model of technological change based upon a simple set of assumptions, which was validated with a large amount of data and could be widely used. The mathematical form is

$$
f=\frac{1}{2}\left[1+\tanh a\left(t-t_{0}\right)\right]
$$

where $f$ is the market share ratio of advanced product. $a$ is half the annual fractional growth, which is positively correlated with the degree of scientific and technological progress.

In the model of Figure 1, OP appears on the market firstly. With the continuous improvement of technology, the OP and NP will be sold in the market for a period of time after NP enters the market. The demand of OP will gradually decrease until OP exits from the market.

According to the paper of Luo [11], when there is only OP in the market, the sales of the product is positively correlated with the total demand of the product in the market, and negatively correlated with the price of the product itself. The market sales model of the single-generation product is

$$
D_{1}=m_{1}-k_{1} P_{1},
$$


where $D_{1}$ are the sales of OP, $m_{1}$ is the potential total demand of OP, $P_{1}$ is the price of OP, and $k_{1}$ is the price sensitivity coefficient of itself. The greater the value of $k_{1}$ is, the more the product sales are affected by its own price, and the less the sales are.

According to the literature of Taylor [17], when there are two substitute products in the market, the sales of each kind of product is not only related to its own total potential demand and its own price, but also affected by the price of substitute products. At this point, the static market sales for the two generations products can be written as

$$
D_{1}=a-k_{1} P_{1}+k_{2} P_{2},
$$

and

$$
D_{2}=a-k_{1} P_{2}+k_{2} P_{1},
$$

respectively, where $D_{2}$ are the sales of the NP, and $P_{2}$ is the price of NP, and the potential total demand of OP and NP are both $a . k_{1}$ and $k_{2}$ are the price elasticity coefficients, which are both greater than $0 . k_{2}$ reflects the influence of the price of substitutes on the demand for products. The higher the price of substitutes is, the more sales of products are. Therefore, the price of substitutes is positively correlated with the demand for products. Since the price of the product directly affects the demand for the product, the price of the original product has a great impact on its demand. The price of substitute products indirectly affects the demand for products through the price of original products, so the effect is secondary. Then $k_{1}>k_{2}$.

In the SC model of Figure 1, it is assumed that the total market demand of OP and NP in the supply chain is $m$. Compared with OP, the NP is seen as a new product, so the sales process of NP can be regarded as a new product diffusion. According to Fisher model [2], the market share of the new product is $f$, so the total market demand of NP is $m * f$, and that of OP is $m^{*}(1-f)$. Then, the market demand of OP and the NP are $D_{1}=m(1-f)-k_{1} P_{1}+k_{2} P_{2} \quad, \quad$ and $D_{2}=m f-k_{1} P_{2}+k_{2} P_{1}$, respectively. Therefore, the demand model when the two generations of products exist in one market is:

$$
\left\{\begin{array}{l}
D_{1}=m(1-f)-k_{1} P_{1}+k_{2} P_{2}, \\
D_{2}=m f-k_{1} P_{2}+k_{2} P_{1}, \\
f=\left[1+\tanh a\left(t-t_{0}\right)\right] / 2 .
\end{array}\right.
$$

The current study focuses on the optimal profits of various entities of supply chain [19]. In actual production, entities are parts of a supply chain and their profits are closely related to each other. Based on the simplified supply chain model, the profits of various entities are analyzed, and the objective of the supply chain is to obtain the optimal total profit.

Firstly, consider the profit of the supply chain from OP:

$$
\pi_{1}=\left(P_{1}-c_{1}-c\right) D_{1},
$$

Similarly, the profit of the supply chain from NP is

$$
\pi_{2}=\left(P_{2}-c_{2}-c\right) D_{2} .
$$

When two generations of the product are sold at the same time, the total profit of the supply chain should be the sum of the profits from two generations of the product, namely

$$
\pi=\pi_{1}+\pi_{2}=\left(P_{1}-c_{1}-c\right) D_{1}+\left(P_{2}-c_{2}-c\right) D_{2},
$$

By substituting Equation (6) into Equation (8)

$\pi=\left(P_{1}-c_{1}-c\right)\left[m(1-f)-k_{1} P_{1}+k_{2} P_{2}\right]+\left(P_{2}-c_{2}-c\right)\left(m f-k_{1} P_{2}+k_{2} P_{1}\right)$,

From the economic point of view, we will seek the biggest value of $\pi$ to maximize the supply chain profit.

Theorem 1: When two generations of a product are sold at the same time, the supply chain's profit $\pi$ can reach the only maximum value at the stationary point on the surface of $\pi$ at $P_{1}$ and $P_{2}$, shown in Equation (14).

Proof: It is necessary to find the gradient matrix and the Hessian matrix.

Firstly, the first-order partial derivatives of $\pi$ with respect to $P_{1}$ and $P_{2}$ are

$$
\frac{\partial \pi}{\partial P_{1}}=m(1-f)+2 k_{2} P_{2}-2 k_{1} P_{1}+k_{1} c_{1}+k_{1} c-k_{2} c_{2}-k_{2} c
$$

and

$$
\frac{\partial \pi}{\partial P_{2}}=m f+2 k_{2} P_{1}-2 k_{1} P_{2}+k_{1} c_{2}+k_{1} c-k_{2} c_{1}-k_{2} c
$$

respectively. Also, the four second-order partial derivatives are

$$
\begin{aligned}
& \frac{\partial^{2} \pi}{\partial P_{1}^{2}}=-2 k_{1}, \frac{\partial^{2} \pi}{\partial P_{1} \partial P_{2}}=2 k_{2}, \\
& \frac{\partial^{2} \pi}{\partial P_{2}^{2}}=-2 k_{1}, \frac{\partial^{2} \pi}{\partial P_{2} \partial P_{1}}=2 k_{2},
\end{aligned}
$$

So, the gradient matrix of $\pi$ is

$$
\nabla \pi\left(P_{1}, P_{2}\right)=\left[\begin{array}{l}
\frac{\partial \pi}{\partial P_{1}} \\
\frac{\partial \pi}{\partial P_{2}}
\end{array}\right],
$$

and the Hessian matrix of $\pi$ is

$$
\nabla^{2} \pi\left(P_{1}, P_{2}\right)=\left[\begin{array}{cc}
\frac{\partial^{2} \pi}{\partial P_{1}^{2}} & \frac{\partial^{2} \pi}{\partial P_{1} \partial P_{2}} \\
\frac{\partial^{2} \pi}{\partial P_{2} \partial P_{1}} & \frac{\partial^{2} \pi}{\partial P_{2}^{2}}
\end{array}\right]=\left[\begin{array}{cc}
-2 k_{1} & 2 k_{2} \\
2 k_{2} & -2 k_{1}
\end{array}\right]
$$

Because $k_{1}$ and $k_{2}$ are always greater than 0 , and $k_{1}>k_{2}, \nabla^{2} \pi$ is a negative definite matrix, and the unique maximum value of $\pi$ can be found. From $\nabla \pi\left(P_{1}, P_{2}\right)=[0,0]^{T}$, the unique stationary point can be got: 


$$
\left\{\begin{array}{l}
P_{1}=\frac{k_{1} m}{2\left(k_{1}^{2}-k_{2}^{2}\right)}-\frac{m f}{2\left(k_{1}+k_{2}\right)}+\frac{c_{1}+c}{2}, \\
P_{2}=\frac{k_{2} m}{2\left(k_{1}^{2}-k_{2}^{2}\right)}+\frac{m f}{2\left(k_{1}+k_{2}\right)}+\frac{c_{1}+c}{2} .
\end{array}\right.
$$

Therefore, the supply chain's profit $\pi$ can reach the only maximum value at the stationary point on the surface of $\pi$ at $P_{1}$ and $P_{2}$ in Equation (14). The proof is finished.

If substituting Equation (14) into Equation (9), the optimal profit can be got as

$$
\pi=\frac{1}{4}\left[\frac{k_{1} m^{2}}{k_{1}^{2}-k_{2}^{2}}+\frac{2 m^{2} f(f-1)}{k_{1}+k_{2}}-2 m f\left(c_{2}+c\right)+k_{1}\left(c_{2}^{2}-c_{1}^{2}\right)+2 k_{1} c\left(c_{2}-c_{1}\right)\right],
$$

Then the cumulative profit of the supply chain from 0 to $t$ is

$$
\Pi=\int \pi d t=\frac{1}{4}\left[\left(\frac{k_{1} m^{2}}{k_{1}^{2}-k_{2}^{2}}+k_{1}\left(c_{2}^{2}-c_{1}^{2}\right)+2 k_{1} c\left(c_{2}-c_{1}\right)-2 m\left(c_{2}+c\right)\right) t+2 m\left(c_{2}+c\right) \ln \left(\tanh \frac{t}{2}+1\right)+\frac{m^{2}}{k_{1}+k_{2}} \tanh \frac{t}{2}\right] .
$$

\subsection{Single-generation Product Sales Model}

From Equation (6), when two generations of a product are sold in market at the same time, the sales of the products are affected by the following three factors: the price of product, the price of the competitive product, and the innovation degree If only NP is sold in the market, the sales of the NP are only affected by its own price and the degree of innovation. At a certain moment, the degree of innovation may be considered unchanged. Therefore, according to the papers of Luo [11] and JC Fisher [2], we can get sales model of NP in the market is

$$
D_{2(o n l y)}=m f-k_{3} P_{2(o n l y)},
$$

where $D_{2}$ (only) are the sales of NP in the market, and $k_{3}$ is the market demand sensitivity to its own price, and it is less than $k_{2}$. Because there is only NP in the market, the potential market demand remains unchanged, and there is no alternative product competing with it, so $k_{2}$ is larger than $k_{1}$, then $0<k_{3}<k_{2}<k_{1}$.

The profit of the supply chain only from NP is

$$
\pi_{2(\text { only })}=\left(P_{2(\text { only })}-c_{2}-c\right) D_{2(\text { only })} .
$$

By substituting Equation (17) into Equation (18), the supply chain's profit function becomes

$$
\begin{array}{r}
\pi_{2(\text { only })}=\left(P_{2(o n l y)}-c_{2}-c\right)\left(m f-k_{3} P_{2(o n l y)}\right) . \quad \text { (19) function of the supply chain become } \\
\pi_{2(o n l y)}=\left(m f-k_{3} P_{2(o n l y)}\right)\left(P_{2(o n l y)}-c_{2}-c\right)=\frac{1}{4}\left[\frac{m f}{\sqrt{k_{3}}}-\sqrt{k_{3}}\left(c_{2}+c\right)\right]^{2} .
\end{array}
$$

Then let the cumulative profit of the supply chain from 0 to $t$ be

$$
\int_{0}^{t} \pi_{2(\text { only })}(t) d t=\frac{1}{4}\left\{\left[\frac{m^{2}}{k_{3}}-2 m k_{3}\left(c+c_{2}\right)^{2}\right] t+\left[\frac{m^{2}}{2 a k_{3}}+\frac{m\left(c+c_{2}\right)}{a}\right] \ln (\tanh a t+1)+\frac{m^{2}}{4 a k_{3}} \tanh a t\right\} .
$$




\section{Optimal Exiting Decision}

\subsection{Exiting Time and Profit}

It is generally believed that a normal time for a product to exit the market is when it is no longer profitable, so we call this time is $t_{1}$. But it may be not the optimal time. Because the cost of OP can no longer be reduced, if it still exists in the market, OP cannot increase its sales and profit. As a result, the supply chain will lose money. Therefore, it is necessary to consider the optimal exit time of OP to maximize the profit of the supply chain.

By modeling and analyzing the sales of two generations of a product (NP and OP) in market at the same time and that of the single-generation product (only NP), it can be known that $\pi$ is the total profit of two generations products and $\pi_{2 \text { (only) }}$ is the profit of NP. When two generations products are sold in the market at the same time, they share the market, and the profit of the supply chain is the sum of the profits of the two products. When only NP is sold in the market, the NP completely monopolizes the market. When there are two generations of a product in market, they will compete inevitably, so the growth

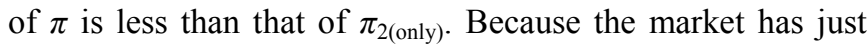
introduced NP, the profit of NP is almost zero at the initial stage. But when there are OP and NP at the market, OP has a certain market status and share, so $\pi$ is far greater than zero. Therefore, usually there must be a time that $\pi$ is equal to $\pi_{2 \text { (only), }}$, named as $t_{2}$. The general trend looks like the following:

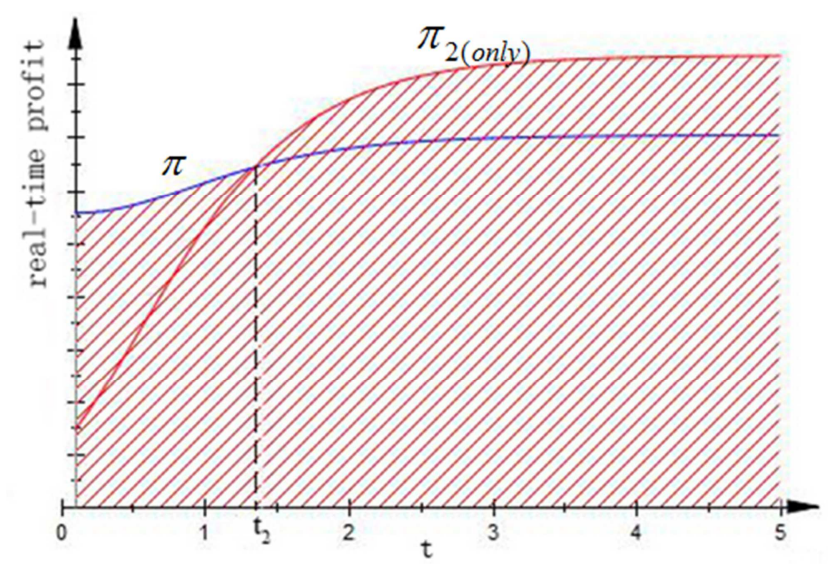

Figure 2. The $O P$ exit from market at $t_{2}$.

From the knowledge of calculus, when OP exits at $t_{2}$, the shaded area is the total cumulative profits of the supply chain from the two generations of a product. It can be calculated by equation (24).

$$
\Pi_{2}=\int_{0}^{t_{2}} \pi d t+\int_{t_{2}}^{t} \pi_{2(o n l y)} d t
$$

Due to the complexity and uncertainties of the market, the exit point $t_{1}$ which is easy to understand may be greater than $t_{2}$, or may be less than $t_{2}$. If OP exits at $t_{1}$, the accumulated profits can be calculated by equation (25).

$$
\Pi_{1}=\int_{0}^{t_{1}} \pi d t+\int_{t_{1}}^{t} \pi_{2} d t
$$

There are two situations:

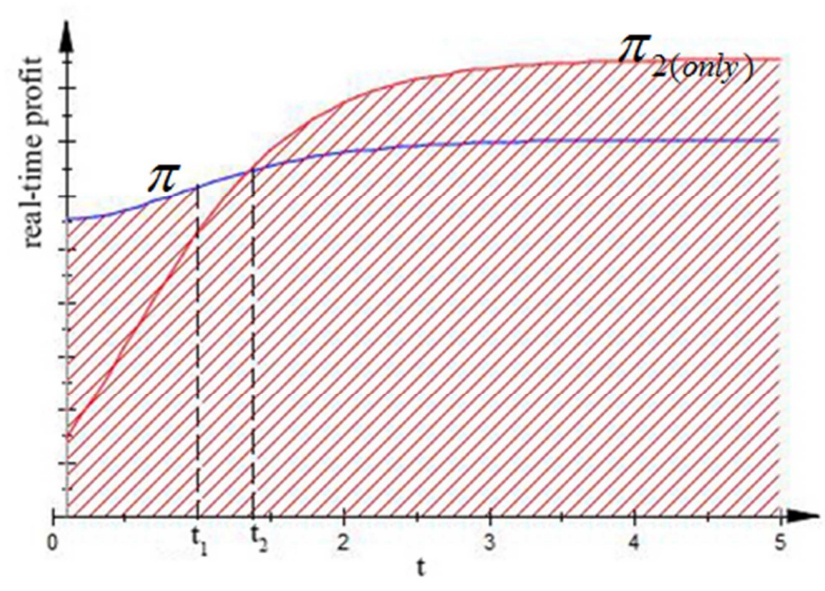

$$
\text { a } t_{1}<t_{2} \text {. }
$$

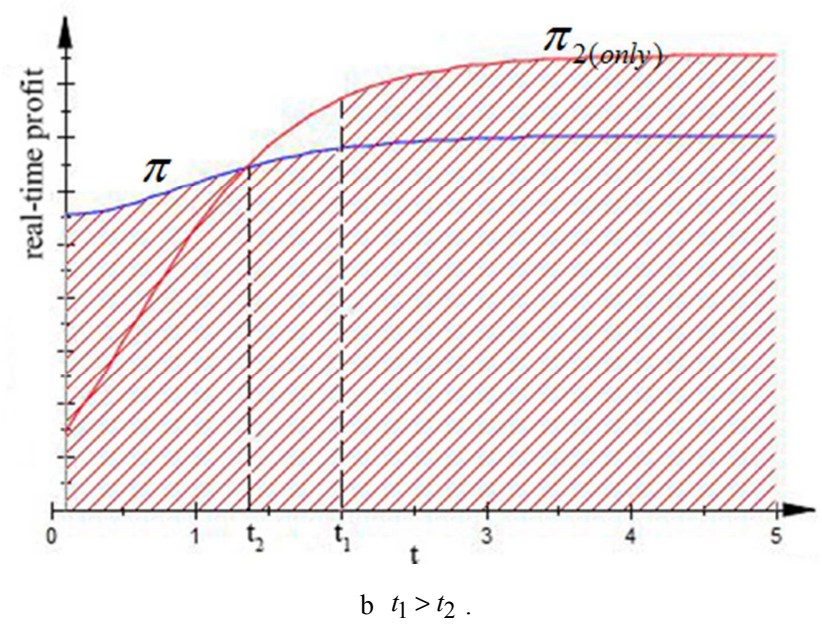

Figure 3. Supply chain's profit considerate exit at $t_{l}$.

OP exit the market at $t_{1}$, if $t_{1}$ is less than $t_{2}$, the supply chain's profits from the two generations of a product are shown in Figure 3.a; if $t_{1}$ is greater than $t_{2}$, The profits of the supply chain are shown in Figure 3.b. Comparing the shaded parts of Figure 2 and Figure 3, we can see that the shaded area of Figure 2 is the largest, which means that SC can get more profits at $t_{2}$ to choose exit OP.

It is not important the value of $t_{1}$, so we just calculate $t_{2}$. According to equation (9) and (19), $t_{2}$ can be calculated as follows.

Making $\pi=\pi_{2 \text { (only) }}$, then:

$$
t_{2}=-\ln \left(-\frac{c k_{3}-m+c_{2} k_{3}}{c k_{3}+c_{2} k_{3}}\right) .
$$

The validity of the exit time is based on the principle that the time value is non-negative.

Theorem 3: When $\exists c>0, k_{3}>0, c_{2}>c_{1}>0$, and meet inequality $k_{3}\left(c_{2}+c\right)<m<2 k_{3}\left(c_{2}+c\right), t_{2}$ is valid.

proof:

According to the principle that the time value is 
non-negative, $\quad t_{2} \geq 0 \quad, \quad$ and $\quad \ln \left(-\frac{c k_{3}-m+c_{2} k_{3}}{c k_{3}+c_{2} k_{3}}\right)<0 \quad$, $-\frac{c k_{3}-m+c_{2} k_{3}}{c k_{3}+c_{2} k_{3}} \in(0,1)$. Let $h=\frac{c k_{3}-m+c_{2} k_{3}}{c k_{3}+c_{2} k_{3}}=\frac{m}{\left.\left(c+c_{2}\right) k_{3}\right)}-1$, and we can have

$$
\left\{\begin{array}{l}
h=\frac{m}{\left.\left(c+c_{2}\right) k_{3}\right)}-1>0, \\
h=\frac{m}{\left.\left(c+c_{2}\right) k_{3}\right)}-1<0,
\end{array}\right.
$$

Therefore, $k_{3}\left(c_{2}+c\right)<m<2 k_{3}\left(c_{2}+c\right)$ is the condition of $t_{2} \geq 0$.

Theorem 4: OP exits at $t_{2}$, then the supply chain can get the maximum profit.

Proof:

First, suppose $\tau$ is the optimal time for OP to exit from market. During the time period $\left(t_{0}, \tau\right), \mathrm{OP}$ and NP are sold at the same time; during $\left(\tau, t_{\infty}\right)$, only NP are sold. $t_{0}$ is the time for NP to enter the market, generally considered to be $0, t_{\infty}$ is the sale time deadline. According to Fisher model, the cumulative profits of the supply chain between $\left(0, t_{\infty}\right)$ are

$$
\Pi=\int_{0}^{\tau} \pi(t) d t+\int_{\tau}^{t_{\infty}} \pi_{2(o n l y)}(t) d t
$$

According to the extreme value theorem, let $\frac{\partial \Pi}{\partial \tau}=0$, we can get $\pi(\tau)=\pi_{2(\text { only) }}(\tau)$, and $\tau$ is effective, then

$$
\tau=-\ln \left(-\frac{c k_{3}-m+c_{2} k_{3}}{c k_{3}+c_{2} k_{3}}\right)
$$

Easy to get $\tau=t_{\text {, }}$, so that $\tau$ or $t_{2}$ is the best time to exit OP.

\subsection{Optimal Exiting Conditions}

Theorem 5: If Condition i): The supply chain adopts the above optimal pricing strategies shown in Equations (14) and (16), and Condition ii): meets inequality $2 m f>\max \left\{\left[m+\left(c_{2}+c\right)\left(k_{1}+k_{2}\right)\right],\left[2 k_{3}\left(c_{2}+c\right)\right]\right\}$, and Condition iii) meets inequalities

$$
\begin{cases}\frac{k_{1} m^{2}}{k_{1}^{2}-k_{2}^{2}}+\frac{2 m^{2} f(f-1)}{k_{1}+k_{2}}-\frac{m^{2} f^{2}}{k_{3}}+k_{1}\left(c_{2}^{2}-c_{1}^{2}\right)+2 k_{1} c\left(c_{2}-c_{1}\right)-k_{3}\left(c_{2}+c\right)^{2}>0, & 0<t<t_{2} \\ \frac{k_{1} m^{2}}{k_{1}^{2}-k_{2}^{2}}+\frac{2 m^{2} f(f-1)}{k_{1}+k_{2}}-\frac{m^{2} f^{2}}{k_{3}}+k_{1}\left(c_{2}^{2}-c_{1}^{2}\right)+2 k_{1} c\left(c_{2}-c_{1}\right)-k_{3}\left(c_{2}+c\right)^{2}>0, & t_{2}<t<\infty\end{cases}
$$

respectively, the optimal exit time for OP is $t_{2}$.

Proof:

To satisfy that $\pi$ and $\pi_{2 \text { (only) }}$ are monotonic increase functions and they have only one intersection in the time period $\left(0, t_{\infty}\right)$, two necessary conditions must be satisfied, which are the condition ii) and iii) in Theorem 5.

The condition ii) is that the first-order differentials of $\pi$ and $\pi_{2 \text { (only) }}$ with respect to $t$ are both greater than 0 .

According to Equations (15) and (17), the first-order derivatives of $\pi$ and $\pi_{2 \text { (only) }}$ with respect to $t$ are

$$
\begin{aligned}
& \frac{\partial \pi}{\partial t}=\left[\frac{m^{2}}{2\left(k_{1}+k_{2}\right)}(2 f-1)-\frac{m\left(c_{2}+c\right)}{2}\right] \frac{\partial f}{\partial t}, \\
& \frac{k_{1} m^{2}}{k_{1}^{2}-k_{2}^{2}}+\frac{2 m^{2} f(f-1)}{k_{1}+k_{2}}-\frac{m^{2} f^{2}}{k_{3}}+k_{1}\left(c_{2}^{2}-c_{1}^{2}\right)+2 k_{1} c\left(c_{2}-c_{1}\right)-k_{3}\left(c_{2}+c_{1}\right)^{2}>0 .
\end{aligned}
$$

$$
\frac{\partial \pi_{2(o n l y)}}{\partial t}=\left[\frac{m^{2} f}{2 k_{3}}-\frac{m\left(c_{2}+c\right)}{2}\right] \frac{\partial f}{\partial t} .
$$

Let the first-order derivatives of $\pi$ and $\pi_{2 \text { (only) }}$ be greater than 0 , we can get $2 m f>\max \left\{\left[m+\left(c_{2}+c\right)\left(k_{1}+k_{2}\right)\right],\left[2 k_{3}\left(c_{2}+c\right)\right]\right\}$. If the above inequality is met, it can be sure that $\pi$ and $\pi_{2 \text { (only) }}$ are monotonic increase functions.

The condition iii) requires that if $t$ is within the scope of

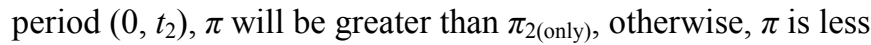
than $\pi_{2(\text { only). }}$ There are two situations are considered as follows:

Situation a) If $t$ is within the scope of period $\left(0, t_{2}\right), \pi$ will be

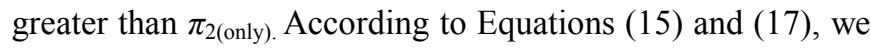

Situation b) If $t$ is within the scope of $\left(t_{2}, \infty\right), \pi$ will be less than $\pi_{2 \text { (only) }}$, and we can get

$$
\frac{k_{1} m^{2}}{k_{1}^{2}-k_{2}^{2}}+\frac{2 m^{2} f(f-1)}{k_{1}+k_{2}}-\frac{m^{2} f^{2}}{k_{3}}+k_{1}\left(c_{2}^{2}-c_{1}^{2}\right)+2 k_{1} c\left(c_{2}-c_{1}\right)-k_{3}\left(c_{2}+c_{1}\right)^{2}>0 .
$$

Equations (32) and (33) can make sure $\pi$ and $\pi_{2 \text { (only) }}$ have only one intersection at time $t_{2}$.

\section{Numerical Simulation}

Considering a cell phone sale market, there are old and new generations of a product in the market and their marginal producing costs are $c_{1}=2000$ and $c_{2}=2300$, respectively, reflecting the higher manufacturing cost of NP than that of OP. The marginal sale cost of the retailer is $c=100$. The total demand of these products is $m=1000$. The current time is $t_{0}=0$. Based on this paper's model, the following simulations were conducted on the products' selling prices and supply chain profit. 


\subsection{Price Analysis}

With the innovation degree of NP, $a$, varying, the pricing strategies of the OP and NP, $P_{1}$ and $P_{2}$, are obtained from Equation (14), as shown in Figures 4 and 5.

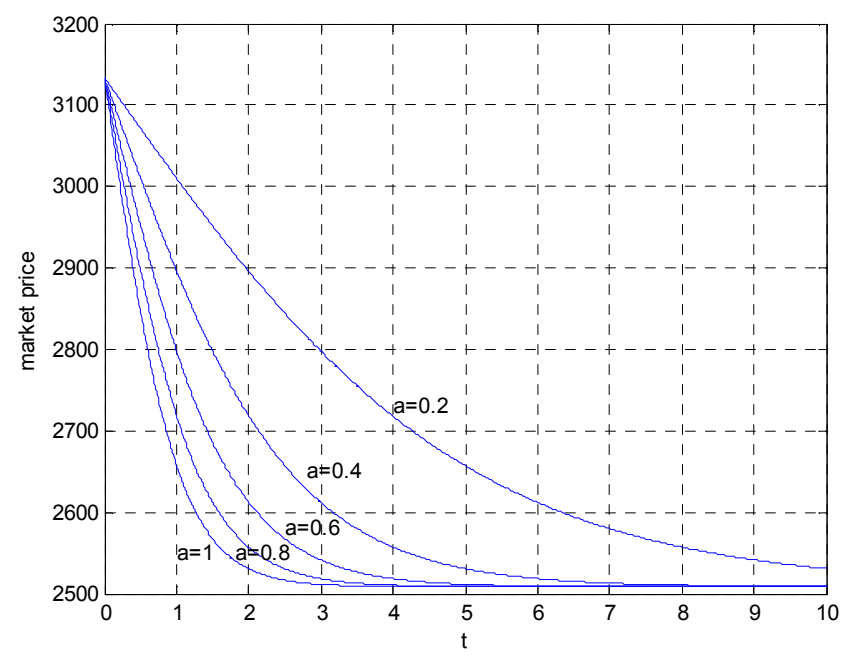

Figure 4. Curves of $P_{1}$ with a varying.

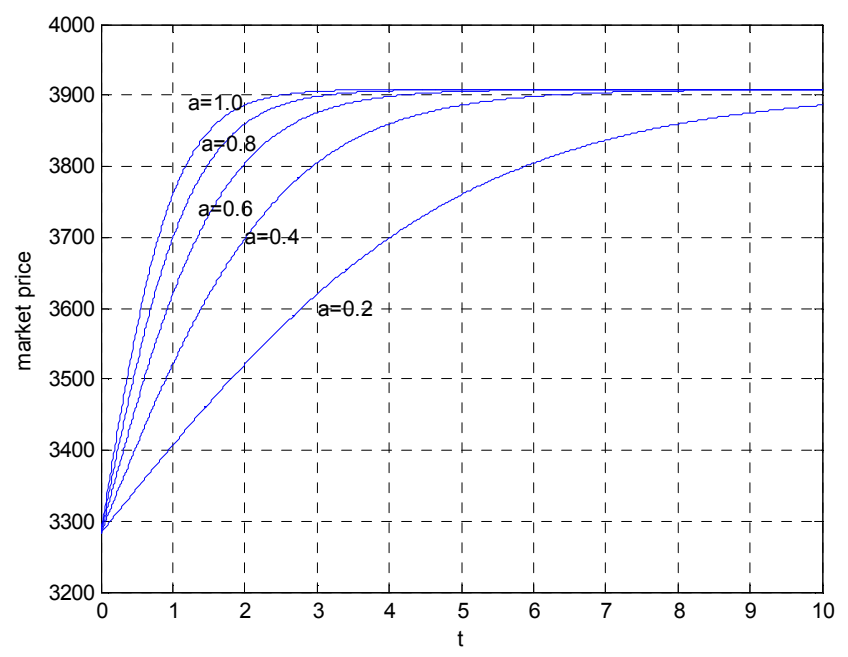

Figure 5. Curves of $P_{2}$ with a varying.

From Figures 4 and 5, two observations can be got: (1) With the innovation degree $a$ varying, the change trends of the price curves are different: with $a$ increasing, the price of OP declines faster and that of the NP increases faster at the beginning. (2) The price curves of the OP decline, converging to the same point near $P_{1}=2500$, while the price curves of the NP increase, converging to the same point near $P_{2}=5700$ regardless of the innovation rate.

This trend meets reality considering the cost of product. Even if OP exit from supply chain, the market price of OP will not lower without lower limit. Considering the market price of NP, even the NP has more innovation and superiority, its price will raise and verge to an upper limit.

When only NP in the market, the curves trend of $P_{2 \text { (only) }}$ is similar to that of $P_{2}$. Set $a=0.5$, the prices strategies are shown in Figure 6.

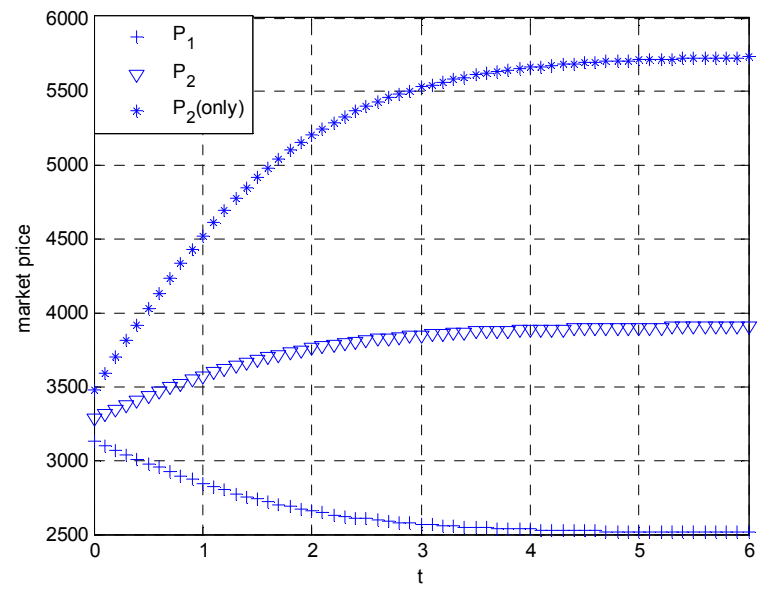

Figure 6. Market price changes with $t$ if $a=0.5$.

\subsection{Sales Analysis}

With the innovation degree $a$ varying, the sales of two generations of a product, $D_{1}$ and $D_{2}$, are obtained from Equation (6), as shown in Figures 7 and 8.

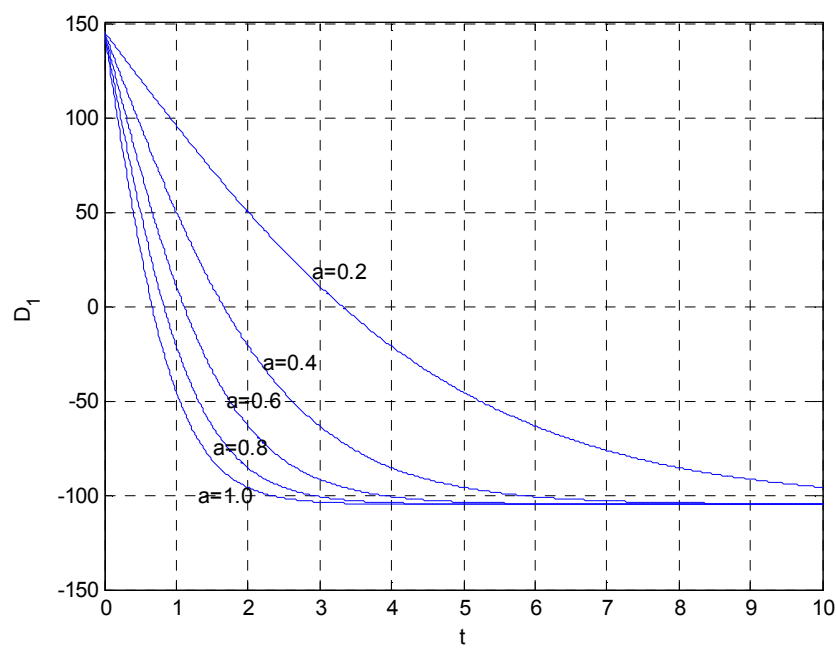

Figure 7. Curves of $D_{l}$ with a varying.

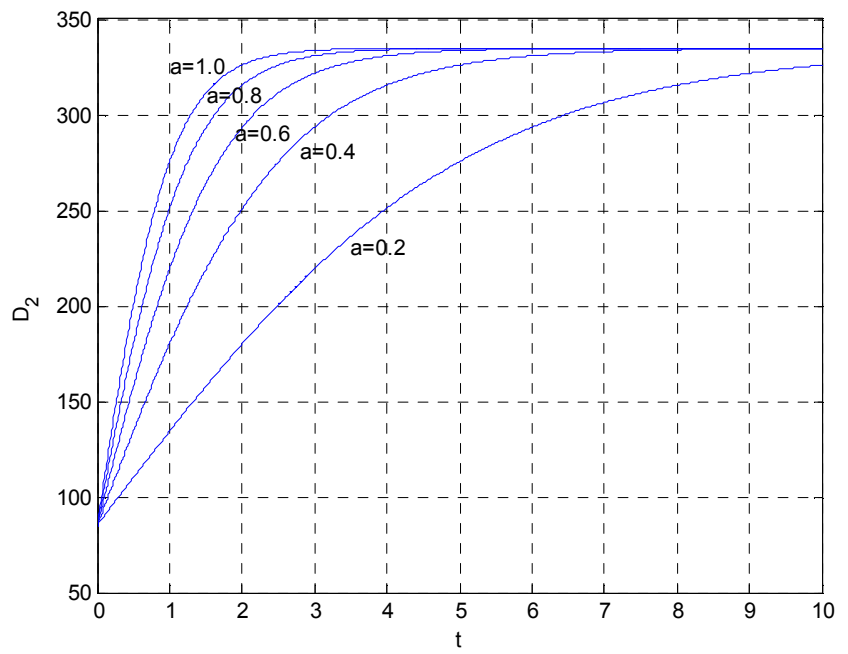

Figure 8. Curves of $D_{2}$ with a varying. 
From Figures 7 and 8, two observations can be got: (1) With the innovation degree $a$ varying, the change trends of the sales curves are different: with $a$ increasing, the sales of the OP declines faster and that of the NP increases faster at the beginning. (2) The sales curves of the OP decline while the sales curves of the NP increase.

When only OP in market, the curves trend of $D_{2 \text { (only) }}$ is similar to that of $D_{2}$. Set $a=0.5$, the demand curves are shown in Figure 9.

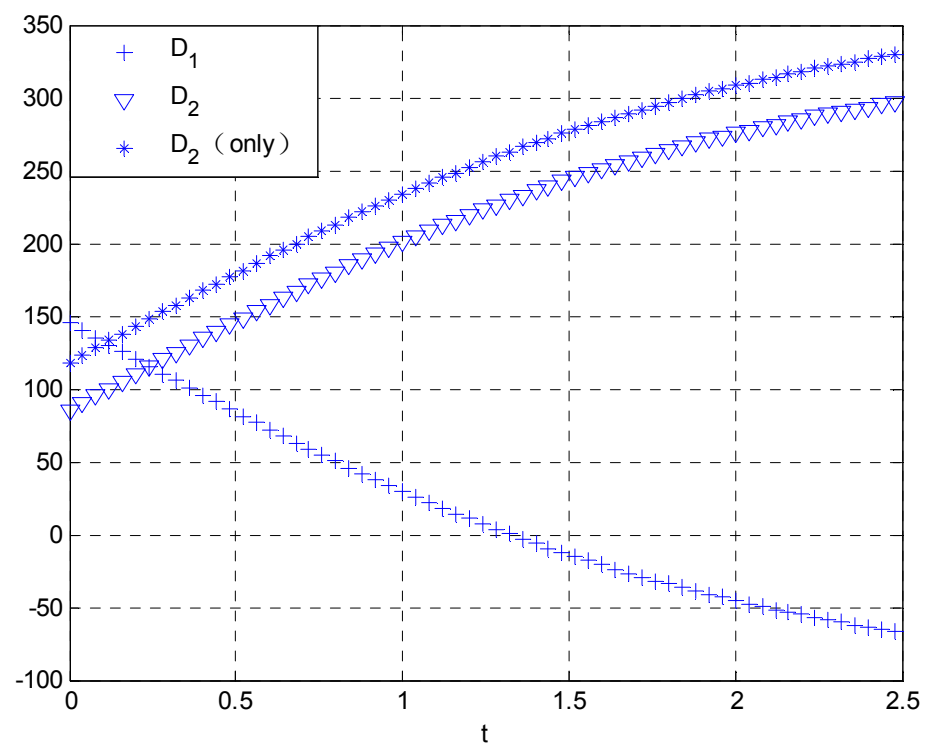

Figure 9. Market sales when $a=0.5$.

\subsection{Exit Time Analyses}

With the exit time of OP varying, the profit of the supply chain is changing. In Table $1, t_{\mathrm{i}}$ is the exit time based on
Equation (26). Parameter $t_{\mathrm{ij}}$, near $t_{\mathrm{i}}$, is the exit time chosen at random. And $t_{\mathrm{i}}$ and $t_{\mathrm{ij}}$ meet the same market conditions. The accumulated profits of SC are shown in Table 1.

Table 1. Accumulated profit comparison.

\begin{tabular}{llllllll}
\hline \multicolumn{2}{l}{ Exit time } & Accumulated profit & Optimal? & Exit time & & Accumulated profit & Optimal? \\
\hline$t_{11}$ & 3.0000 & 6790289 & no & $t_{21}$ & 1.0000 & 9213350 & no \\
$t_{12}$ & 5.2000 & 7151026 & no & $t_{22}$ & 1.2000 & 9236843 & no \\
$t_{1}$ & 5.3917 & 7521855 & yes & $t_{2}$ & 1.3479 & 9241594 & yes \\
$t_{13}$ & 5.5000 & 7152350 & no & $t_{23}$ & 1.5000 & 9237106 & no \\
$t_{14}$ & 6.0000 & 7134995 & no & $t_{24}$ & 3.0000 & 8932263 & no \\
\hline
\end{tabular}

From Table 1, when $t_{1}$ and $t_{2}$ are exit times, respectively, the supply chain can get more profit. Therefore, it can be considered that the exit time proposed in this paper is the optimal exit time for OP.

With the innovation degree $a$ varying, the exit time of OP is changing, and with the exit time varying, the profit of the supply chain is changing, too. The relationships among $a$, the exit time and the profit are shown in Table 2.

Table 2. Relationship among $a$, exit time and profit.

\begin{tabular}{|c|c|c|c|c|c|c|}
\hline \multirow{2}{*}{$\mathbf{a}$} & \multicolumn{2}{|c|}{ Exit time } & \multicolumn{3}{|c|}{ Accumulated profit } & \multirow{2}{*}{$\begin{array}{l}\text { Optimal exit time } \\
t_{2}\end{array}$} \\
\hline & $t_{1}$ & $t_{2}$ & not consider exit & Exit at $t_{1}$ & Exit at $t_{2}$ & \\
\hline 0.2 & 5.9730 & 5.3917 & 7241189 & 7349924 & 7521855 & $\mathrm{t}_{2}$ \\
\hline 0.4 & 2.9865 & 2.6958 & 7664397 & 7850148 & 8283059 & $t_{2}$ \\
\hline 0.6 & 1.9909 & 1.7972 & 7914801 & 8231641 & 8909832 & $\mathrm{t}_{2}$ \\
\hline 0.8 & 1.4932 & 1.3479 & 8045561 & 8432739 & 9241594 & $\mathrm{t}_{2}$ \\
\hline 1.0 & 1.1946 & 1.0783 & 8124425 & 8921796 & 9441994 & $t_{2}$ \\
\hline
\end{tabular}

From Table 2, two observations can be got: (1) The relationships among $a$, exit time and profit are: the higher innovation degree is, the earlier the old generation product exits the market, the more profit the $\mathrm{SC}$ will get no matter what exit strategies the supply chain adopts. (2) It can be seen from the column of cumulative profit that the supply chain's profit with an exit strategy are higher than without.

When $a=0.5$, for example, the exiting time of the old 
generation product is shown in Figure 10.

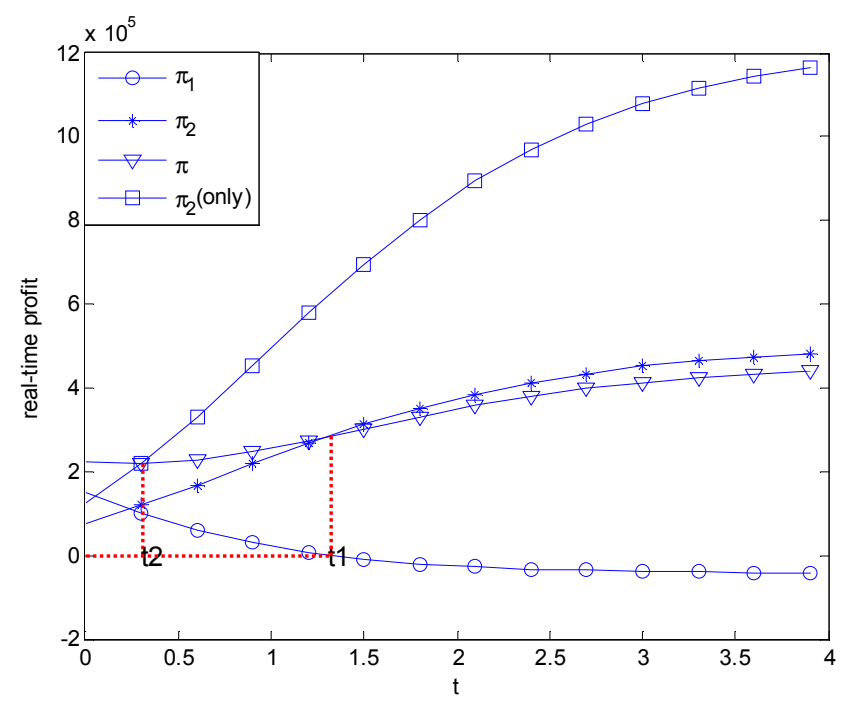

Figure 10. Real-time profits.

At $a=0.5$, according to Equations (16), (24) and (25), the accumulated profits with different exit strategies are shown in Figure 11.

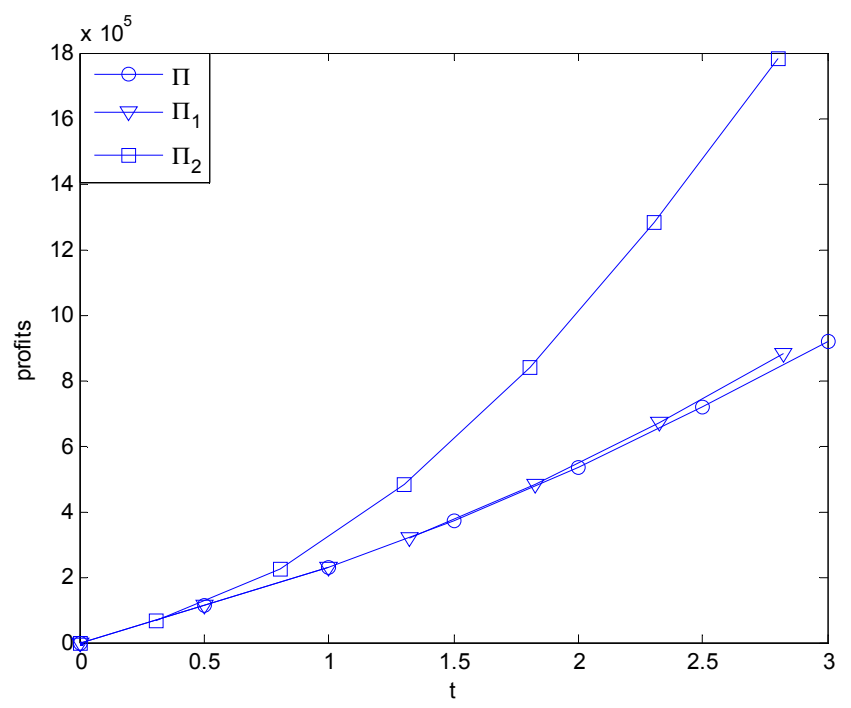

Figure 11. Changes of accumulated optimal profits.

In Figure 11, $\Pi$ is the profit of SC without exit strategy, and $\Pi_{1}, \Pi_{2}$ are the profits of supply chain with an exit strategy at $t_{1}$ and $t_{2}$, respectively. It can be observed that: (1) the profit with an exit strategy is higher than without. (2) $\Pi_{2}$ is the biggest profit, namely, OP exiting at $t_{2}$ proposed by this paper is optimal for the supply chain. The result of Figure11 is consistent with Table 2.

\section{Conclusion}

This paper built new market-demand models for two generations and a upgraded generation of a product based on the Fisher model and multi-generation product diffusion model. The new model described market demand changes for old or new generation product over time. It also established a price making-decision model for supply chain based on the new model. Furthermore, the optimal time for the old product to exit the market has been determined.

Several innovative observations were got: (1) with the exit strategy for the old generation product, the profit of supply chain increases; (2) the change rates of demand and the retailer's price for two generations of a product over time are opposite, too; and (3) with the innovation degree increasing, the time for the old product to exit the market is earlier. Lastly, a numerical study is performed to exemplify the above conclusions. This paper can help decision-makers to determine the prices for upgraded products in a supply chain.

As an initial attempt to build a model based on Fisher model to deal with the exit time of old generation product, the modle proposed by this paper has some limitations. First, the model is simple. The model only considers the two major factors affecting diffusion. The two factors are prices of NP and OP and innovation level of new generation product. However, the market environment is complex, and product diffusion is affected by many factors, such as advertising, promotion and supply chain management. In the future, research on product exiting, these factors can be added to establish a more accurate exiting model and better guide the practice.

\section{Acknowledgements}

This work was supported by the Foundation and research in cutting-edge technologies in the project of Henan province, China [Grant No. 132300410420]; the Young Teacher Foundation of Henan province [Grant No. 2015GGJS-148].

\section{References}

[1] Bass Frank M. A New Growth for Model Consumer Durables [J]. Management Science, 1969, 15(9): 215-227.

[2] J. C. Fisher, R. H. Pry. A Simple Substitution Model of Technological Change [J]. Technological forecasting and social change, 1972: 75-88.

[3] Norton J A, Bass F M. Evolution of technological generations: The law og capture [J]. Sloan Management Review, 1992, 33(2): 66-77.

[4] Yong Luo, Fengsheng Tu. Differential Products Combination Pricing Strategy in Supply Chain [J]. International conference on services systems and services management, 2005, 1: $640-646$.

[5] Seref, MMH (Seref, Michelle M. H.), Carrillo, JE (Carrillo, Janice E.); Yenipazarli, A (Yenipazarli, Arda). Multi-generation pricing and timing decisions in NP development [J] International Journal of Production Research, 2016, 54(11):1919-1937.

[6] Matsui, K (Matsui, Kenji). When and what wholesale and retail prices should be set in multi-channel supply chains? [J] European Journal of Operational Research, 2018, 267(6):540-554. 
[7] Udayan Chanda, Remica Aggarwal. Optimal inventory policies for successive generations of a high technology product [J]. Journal of High Technology Management Research, 2014, 25: $148-162$.

[8] Xiongwen Quan, Fengsheng Tu, Jie Wei. The stackelberg game of the price of product between manufacturers and sellers $[\mathrm{J}]$ System engineering theory and practice, 2007, 18(12):111-118.

[9] Gupta, MC (Gupta, Manak C.); Di Benedetto, CA (Di Benedetto, C. Anthony). Optimal pricing and advertising strategy for introducing a new business product with threat of competitive entry [J] Industrial Marketing, 2007, 36(8):540-548.

[10] Cheryl T. Druehl, Glen M. Schmidt, Gilven C. Souza. The optimal pace of product updates [J]. European journal of operational research, 2009, 192: 621-633.

[11] Luo Yong, Lou Yamin, Wang Shizhao. Stackelberg game for product renewal in supply chain [J] Discrete dynamics in nature and society, 2013.

[12] Paraskevas C. Argouslidis, George Baltas, Alexis Mavrommatis. An empirical investigation into the determinants of decision speed in product elimination decision processes [J]. European management journal, 2015, 33: $268-286$.
[13] Wei Shi Lim, Christopher S. Tang. Optimal product rollover strategies [J]. European journal of operational research, 2006, 174: $905-922$.

[14] De Cesare Luigi, Di Liddo Andrea. A stackelberg game of innovation diffusion: Pricing, advertising and subsidy strategies [J]. International Game Theory Review, 2001, 3(4): 325-340.

[15] Shaikh N I, Rangaswa A, Balakrishnan A. Modeling thediffusion of innovations using small-world networks [M]. CBMS Regional Conference, 2006.

[16] Zifang Lu, Ling Zeng. Competitive new product diffusion control $[\mathrm{J}]$. Journal of university of posts and telecommunications of Nanjing, 2017, 37(5): 62-67.

[17] Taylor. Industrial organization theory. Beijing: China renmin university press, 1997:166-200.

[18] Shlomo Kalish. Monopolistic pricing with dynamic demand and production cost [J]. Marketing Science, 1983, 2(6): $135-159$.

[19] Shizhao Wang. Research on optimal decision making of supply chain entity coordination [D]. Henan: Zhengzhou University, 2014. 